\title{
Optimization of Integer Order Integrators for Deriving Improved Models of Their Fractional Counterparts
}

\author{
Maneesha Gupta and Richa Yadav \\ Advanced Electronics Lab, Department of ECE, Netaji Subhas Institute of Technology, Dwarka, New Delhi 110078, India \\ Correspondence should be addressed to Richa Yadav; yadav.richal@gmail.com
}

Received 28 February 2013; Revised 21 May 2013; Accepted 30 May 2013

Academic Editor: Manuel Lozano

Copyright (C) 2013 M. Gupta and R. Yadav. This is an open access article distributed under the Creative Commons Attribution License, which permits unrestricted use, distribution, and reproduction in any medium, provided the original work is properly cited.

Second and third order digital integrators (DIs) have been optimized first using Particle Swarm Optimization (PSO) with minimized error fitness function obtained by registering mean, median, and standard deviation values in different random iterations. Later indirect discretization using Continued Fraction Expansion (CFE) has been used to ascertain a better fitting of proposed integer order optimized DIs into their corresponding fractional counterparts by utilizing their refined properties, now restored in them due to PSO algorithm. Simulation results for the comparisons of the frequency responses of proposed 2nd and 3rd order optimized DIs and proposed discretized mathematical models of half integrators based on them, with their respective existing operators, have been presented. Proposed integer order PSO optimized integrators as well as fractional order integrators (FOIs) have been observed to outperform the existing recently published operators in their respective domains reasonably well in complete range of Nyquist frequency.

\section{Introduction}

In fractional calculus (FC) initially the development of mathematical models of fractional order integrators (FOIs) and fractional order differentiators (FODs) [1, 2] started presumably for searching different generalizing approaches for switching from integer order to the fractional order domain. The frequency response of ideal fractional order differ-integrator $\left(s^{ \pm \alpha}\right)$ is

$$
H_{\text {fractional }}(j \omega)=(j \omega)^{ \pm \alpha},
$$

where $j=\sqrt{-1}$ and $\omega$ gives angular frequency in radians. Variable " $\alpha$ " defines the order of fractional order operators and its value lies in between 0 and 1 .

Due to its exotic nature FC has strengthened its grasp over different research areas such as statistical modeling, mechanical system analysis [3], control [4], automated control [5], instrumentation [6], signal processing [7], and radio engineering and image processing $[8,9]$. At present time, $\mathrm{FC}$ is also extensively spreading its niche in different design methods for improving fractional order controllers by fractional integral and derivative functions $[10,11]$. An important design method which has undoubtedly helped in sharpening up the focus of an efficient formulation of new blocks of fractional operators is "linear interpolation" method [1216]. This most vastly followed tri-step method involves the following steps. (1) First step deals with linear interpolation of two existing digital integrators. (2) In second step, a new fractional order integrator is formulated by discretization of integer order integrator developed in first step by anyone of the two existing discretization (direct and indirect) schemes. (3) In the third step, this expanded series is truncated up to some arbitrary order $(N)$, which defines the order of the new fractional operator $\left(s^{ \pm \alpha}\right)$. Direct discretization [17] works with the help of different expansion techniques such as Taylor Series Expansion (TSE) [18], Power Series Expansion (PSE) [19, 20], and Continued Fraction Expansion (CFE) [21, 22], whereas for indirect discretization [23-26] different rational approximations have been developed by many mathematicians, namely, Carlson and Halijak [27], Steiglitz [28], Khovanskii [29], Roy [30], Oustaloup et al. [31], 
and Maione [32], which proved like boon for the development of fractional integrators/differentiators.

In spite of the efficient and systematic nature of different previously mentioned approximations, still there existed a dissent about performance of these approximations, when these were later adapted by well-known discretization schemes for obtaining FOIs. Moreover development of new approximations required many exhaustive mathematical applications to make them perform in finite dimensional form in proper range of frequencies during discretization. Instead of deriving new methods of rational approximations, authors have suggested here an alternate solution in which the integrator operator (used as $s-z$ transform) itself should be pushed near boundaries of an optimal solution (near ideal response) before converting it to fractional system. Reason behind this is that every minute dispensation in properties of this integer order operator will directly propel into its noninteger order counterpart. The main novelty proposed by this paper is to put together unique combination of optimized integer order operators and an accurate approximation technique for configuring their efficient fractional order (fo) operators. The current scenario of design methods for deriving optimal operators [33-41], is cumulatively proceeding towards "not so distant" phase of perfect optimization with negligible errors. So, another motivating point behind the approach proposed in this paper is to trespass all disadvantages of existing conventional design methods of FOIs by using refined capabilities of an efficient optimization scheme.

The frequency response of ideal integer order digital integrators (DIs) is

$$
H_{\mathrm{int}}(\omega)=\left(\frac{1}{j \omega}\right), \quad \text { where }|\omega| \leq \pi,
$$

and the frequency response of ideal integer order digital differentiators (DDs) is

$$
H_{\text {diff }}(\omega)=j \omega, \quad \text { where }|\omega| \leq \pi,
$$

where $j=\sqrt{-1}, \omega$ is the angular frequency in radians, and " $T$ " is the sampling period.

In recent novel advancements, different optimization algorithms, namely, Linear Programming (LP) [33, 34], Genetic Algorithm (GA) [35-37], Simulated Annealing (SA) $[38,39]$, and pole-zero (PZ) optimization $[40,41]$, have been used for obtaining more refined integer order differintegrators. Jain et al. [35] have recently derived optimal models of recursive DIs and DDs by GA algorithm while Upadhyay [41] has developed these operators by analyzing poles and zeros of existing recursive DDs by $\mathrm{PZ}$ optimization technique for obtaining better results for frequency responses over wideband of complete frequency spectrum. Al-Alaoui [39] has done a credible work in improving design process of DIs and DDs based on Newton-Cotes integration rules by using linear interpolation and SA.

This paper attempts to find the optimal solutions of DIs by using an efficient modified application of Particle Swarm Optimization (PSO) technique [42-44], before discretizing them to their fractional mathematical models.
Values of mean, median, and standard deviation in different random iterations have been continuously registered using PSO for the optimization of 2nd and 3rd order DIs with minimized error fitness function. Later these optimized DIs are discretized by CFE of indirect discretization scheme [23], for finding half integrator models of different orders. The simulation results of magnitude responses, phase responses, and relative magnitude errors for the proposed optimized DIs have been compared with all the recently published integrators optimized by different optimization algorithms, namely, LP [34], GA [35], SA [39] and PZ [41]. Frequency responses of the proposed FOIs based on 2nd and 3rd order optimized DIs have been also compared with their existing fractional models $[2,17]$ in complete frequency range.

The paper is organized as follows. Section 2 deals with the brief description of original and modified PSO algorithm for optimizing DIs along with its trade-off conditions, and the resultant 2 nd and 3rd order optimized integrators thus derived have been presented in this section. Comparisons of proposed and existing DIs are also given in the same section. These optimized operators have been discretized by indirect discretization using CFE technique of indirect discretization for deriving models of FOIs in Section 3. Section 4 presents the simulation results of comparisons of proposed half integrators with responses of ideal and the existing models. Section 5 concludes the paper.

\section{PSO Algorithm for Optimizing 2nd and 3rd Order Integer Order Integrators}

2.1. Outline of Basic Functionality of Original PSO Algorithm. In conventional PSO algorithm [42-44], first a population of random solutions has been initialized and different generations are updated for searching optimal values. In this algorithm, the potential solutions, called particles, fly through the problem space by following the current optimum particles. The position of each particle encodes a possible solution to the given problem. The velocity of the particle is the parameter to be added to the current position to find the new position of the next generation. The velocity and position are updated for all particles in every generation unless an optimal solution is found. The particles keep track of its coordinates in the problem space which are associated with the best solution (fitness) it has achieved so far. These values are called pbest and the best element among all the pbest values of all the particles is called gbest. The velocities are initialized to zero and after finding the two best values, the particle updates its velocity and positions in accordance with the following update equations:

$$
\begin{gathered}
v(i)=v(i-1)+c_{1} r_{1}\left(p_{\text {pbest }}-p(i)\right)+c_{2} r_{2}\left(p_{\text {gbest }}-p(i)\right), \\
p(i+1)=p(i)+v(i),
\end{gathered}
$$

where $v(i)$ is the current particle velocity, $v(i-1)$ is previous particle velocity, $p(i)$ is current particle position, $p(i+1)$ is the position of particle in next generation, $p_{\text {pbest }}$ is the best 


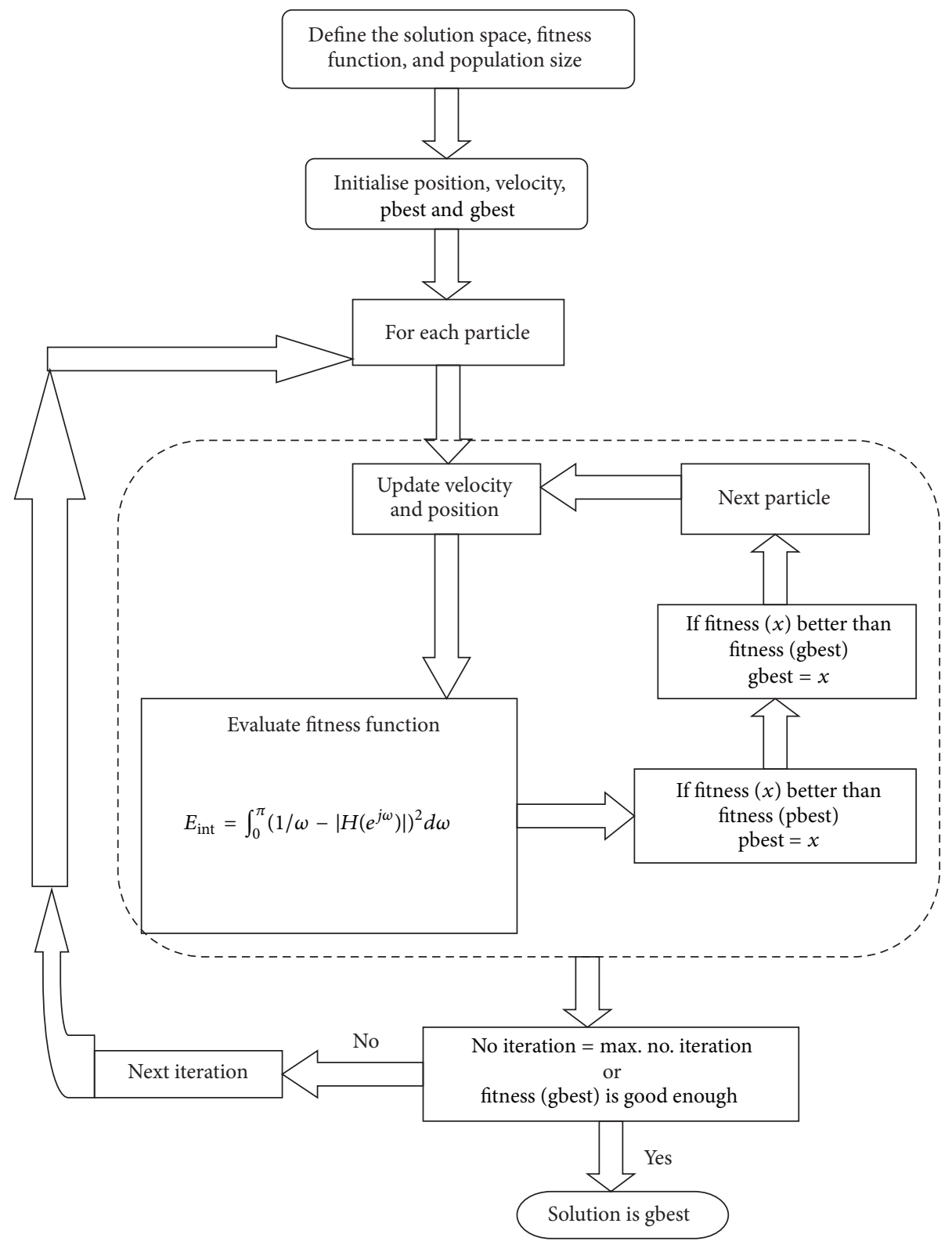

FIGURE 1: Flowchart of modified PSO algorithm.

position the particle has seen, $p_{\text {gbests }}$ is the best position the swarm has seen, $c_{1}, c_{2}$ are constants set to 2 , and $r_{1}, r_{2}$ are the random numbers between 0 and 1 .

\subsection{Parameters Used for Optimization and Trade-Offs between} Multiple Parameters during Convergence of Optimized Digital Integrators. A mathematical model of transfer function (TF) of optimized digital integrator (DI) can be described in generalized form as

$$
H(z)=\left(\frac{B_{M}(z)^{b_{M}}+\cdots+B_{1}(z)^{b_{1}}+B_{0}(z)^{b_{0}}}{A_{N}(z)^{a_{N}}+\cdots+A_{1}(z)^{a_{1}}+A_{0}(z)^{a_{0}}}\right),
$$

where $A_{i}$ and $B_{j}$, are arbitrary constants whereas $a_{i}$ and $b_{j}$ are real numbers with $(i=0,1, \ldots, N)$ and $(j=0,1, \ldots, M)$.
Transfer functions (TFs) of 2nd and 3rd order DIs which have been optimized using PSO in this paper are given in (6) and (7), respectively:

$$
\begin{gathered}
H_{\text {PSO_2nd_opt }}(z)=\left(\frac{B_{2} z^{2}+B_{1} z+B_{0}}{A_{2} z^{2}+A_{1} z+A_{0}}\right), \\
H_{\text {PSO_3rd_opt }}(z)=\left(\frac{B_{3}(z)^{3}+B_{2}(z)^{2}+B_{1} z+B_{0}}{A_{3}(z)^{3}+A_{2}(z)^{2}+A_{1} z+A_{0}}\right) .
\end{gathered}
$$

The coefficients of the previously mentioned TFs are generated by random iterations of PSO algorithm as it takes real numbers as particles. 
TABLE 1: Transfer functions and fitness values of worst and average cases of 2 nd order PSO optimized DIs.

\begin{tabular}{|c|c|c|c|}
\hline \multicolumn{2}{|c|}{$\begin{array}{l}\text { TFs of worst cases of } 2 \text { nd order DIs along with their fitness } \\
\text { function values (F.V.) }\end{array}$} & \multicolumn{2}{|c|}{$\begin{array}{l}\text { TFs of average cases of } 2 \text { nd order DIs along with their fitness } \\
\text { function values (F.V.) }\end{array}$} \\
\hline Transfer function & F.V. & Transfer function & F.V. \\
\hline$H_{1}(z)=\left(\frac{0.373324 z^{2}+0.427523 z+0.397575}{z^{2}-0.042561 z-0.999916}\right)$ & 0.643052 & $H_{1}(z)=\left(\frac{0.22896 z^{2}-0.825829 z-0.502494}{z^{2}-0.776284 z-0.181729}\right)$ & 0.382107 \\
\hline$H_{2}(z)=\left(\frac{-0.929251 z^{2}+0.381076 z-0.75834}{z^{2}-0.725805 z-0.306558}\right)$ & 3.90016 & $H_{2}(z)=\left(\frac{-0.336478 z^{2}-0.82224 z+0.101197}{z^{2}-0.818946 z-0.147828}\right)$ & 0.407093 \\
\hline$H_{3}(z)=\left(\frac{0.541699 z^{2}+0.241831 z+0.506633}{z^{2}-0.0552516 z-1}\right)$ & 4.73234 & $H_{3}(z)=\left(\frac{0.0849835 z^{2}+0.752164 z+0.756218}{z^{2}-0.167219 z-0.882869}\right)$ & 0.50102 \\
\hline$H_{4}(z)=\left(\frac{-0.354583 z^{2}-0.833923 z-0.813869}{z^{2}-0.0637125 z-1}\right)$ & 5.89578 & $H_{4}(z)=\left(\frac{-0.39729 z^{2}-0.795585 z-0.130415}{z^{2}-0.722962 z-0.318656}\right)$ & 0.618717 \\
\hline$H_{5}(z)=\left(\frac{0.353852 z^{2}+0.203374 z+0.466221}{z^{2}-0.0406162 z-1}\right)$ & 18.799 & $H_{5}(z)=\left(\frac{0.284382 z^{2}+0.949776 z+0.363676}{z^{2}-0.401952 z-0.648204}\right)$ & 0.737534 \\
\hline
\end{tabular}

Magnitude response of the optimized DI is

$$
H_{\text {mag }}=|H(w)|=\frac{\text { abs (numerator) }}{\text { abs (denominator) }} .
$$

Phase response of the optimized DI is

$$
H_{\text {phase }}=\arg |H(w)| \text {. }
$$

Relative magnitude error $(\mathrm{dB})$ or RME value is given by

$$
\begin{aligned}
\mathrm{RME} & =20 \log _{10}\left|\frac{H_{\text {ideal }}(\omega)-H_{\text {approx }}(\omega)}{H_{\text {ideal }}(\omega)}\right| \\
& =20 \log _{10}\left|\frac{(1 / j \omega)-H_{\text {approx }}(\omega)}{(1 / j \omega)}\right|,
\end{aligned}
$$

where $H_{\text {ideal }}(\omega)$ is the magnitude of ideal value of the operator and $H_{\text {approx }}(\omega)$ is the value of the digital integrator that is approximated.

In the recently published literature on digital and fractional operators most of the researchers have focused on formulating the fitness function for optimization technique based on only single parameter [38-41] that is magnitude error because practically if phase and magnitude are optimized for a multiparameter problem the solution is always stuck in a trade-off due to their contradictory nature. The reason behind this is that when the optimal solution starts converging towards the best results of one parameter (magnitude response) it simultaneously degrades optimality of second parameter (phase responses) after certain number of iterations. Solution for such trade-offs is to choose dominating parameter in problem under consideration. In this paper the design strategy to find optimized DIs is kept more concerned for minimum magnitude response error, which is comparatively more significant characteristic. The fitness function used to approximate a digital integrator is taken as the mean square error of the transfer function and it is given as

$$
E_{\text {int }}=\int_{0}^{\pi}\left(\frac{1}{\omega}-\left|H\left(e^{j \omega}\right)\right|\right)^{2} d \omega .
$$

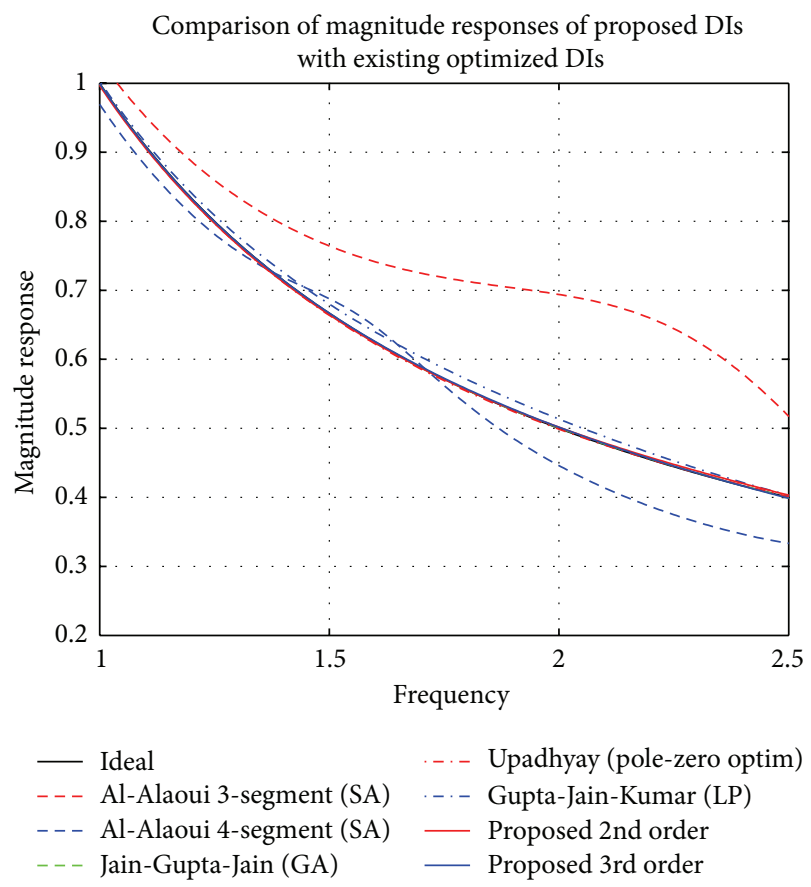

FIGURE 2: Comparison of magnitude responses of proposed 2nd and 3rd order optimized DIs with existing [26, 34, 39, 41] optimized models.

Aim of PSO optimization is to search the best optimal solution (TF of DI) with minimized mean square error.

\subsection{Modified PSO Algorithm for Derivation of Optimized} DIs. In this paper, a slight modification in the original PSO algorithm has been introduced in velocity, taking into account the given problem of deriving the best feasible optimized DIs with very small magnitude response errors without worsening their corresponding phase responses. In modified PSO used in this paper, the maximum velocity which constraints the movement of swarms to the range is 


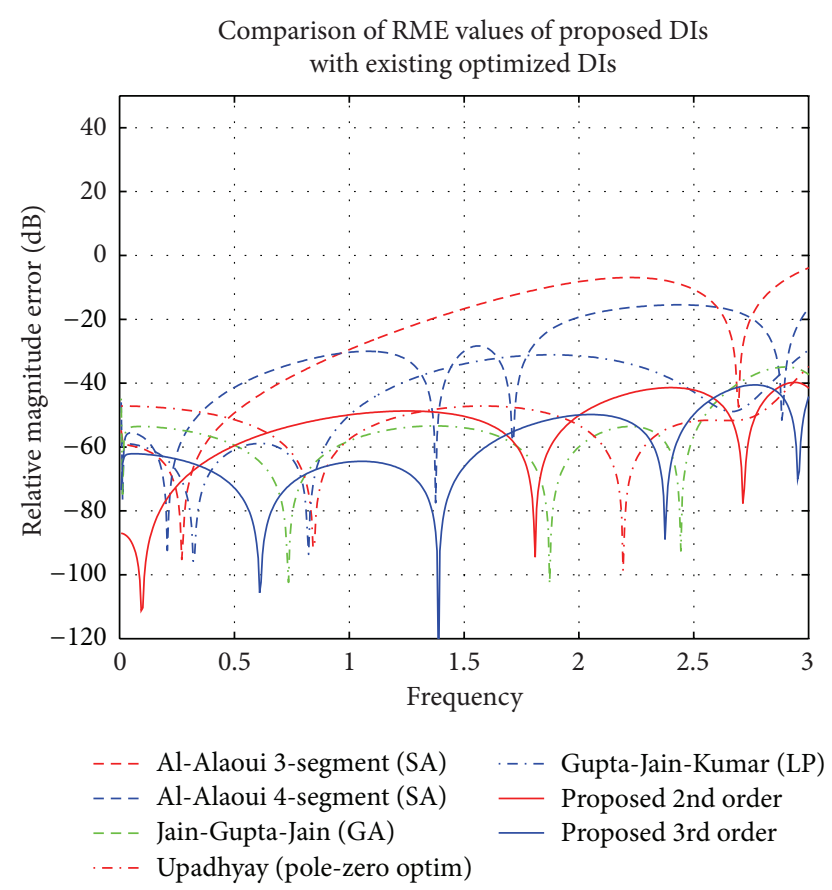

Figure 3: Comparison of RME values of proposed 2nd and 3rd order optimized DIs with existing $[26,34,39,41]$ optimized models.

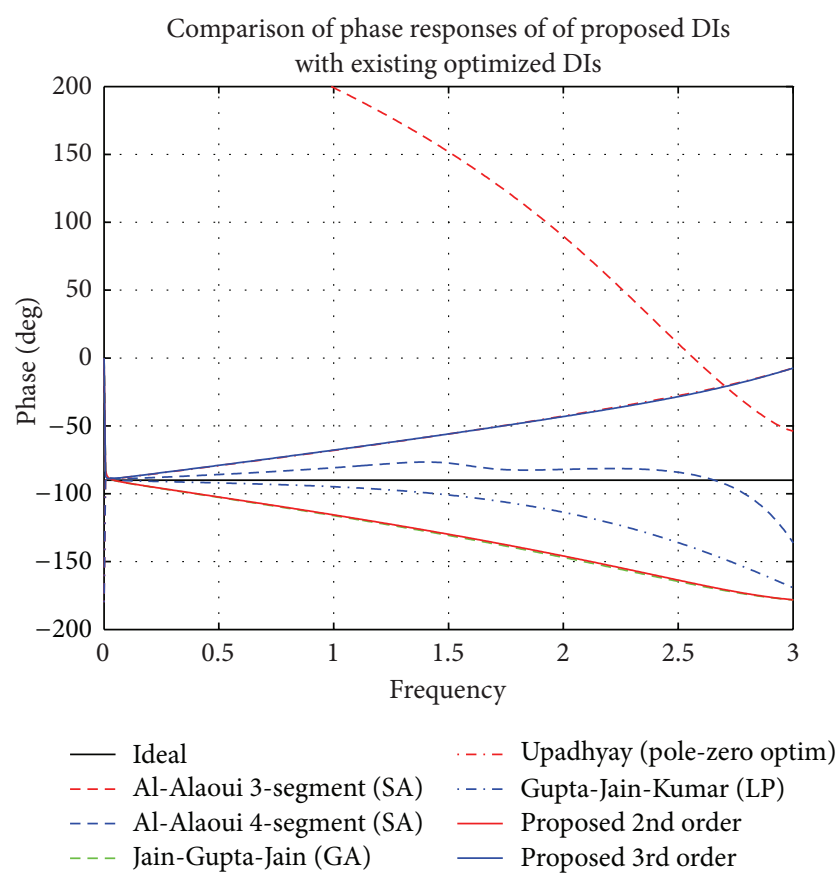

FIGURE 4: Comparison of phase responses of proposed 2nd and 3rd order optimized DIs with existing [26, 34, 39, 41] optimized models.

divided by a factor of ten after a certain number of iterations. Here we have used dynamic velocity control variant of PSO in which velocity is scaled down after every specific number of generations. This modification results in improved performance as compared to the original algorithm. In this paper the parameters used for PSO algorithm for finding

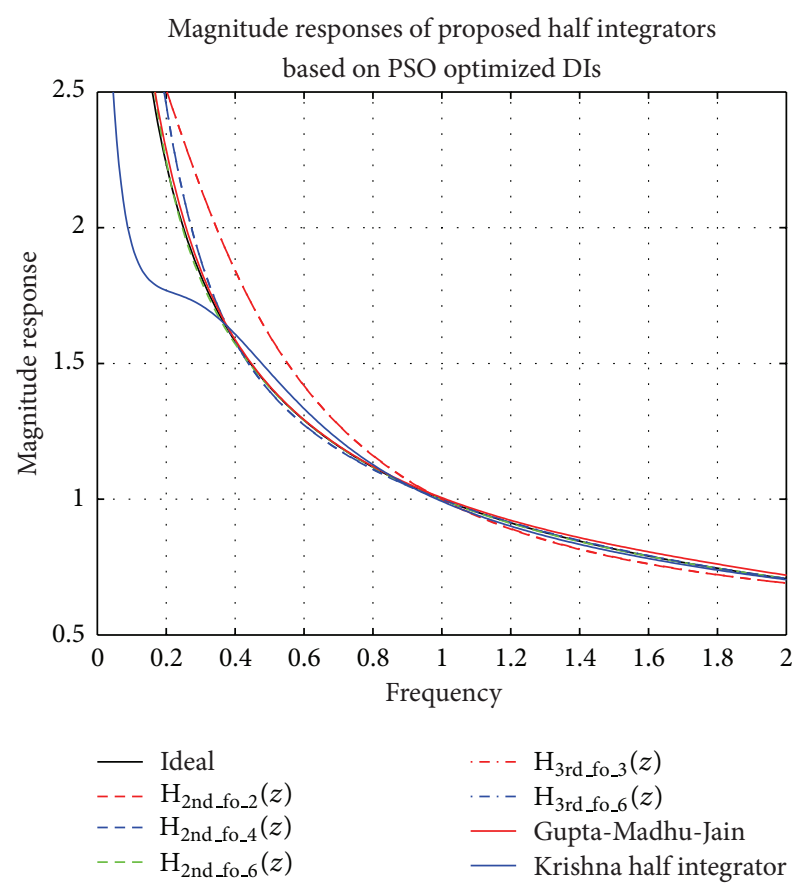

Figure 5: Comparison of magnitude responses of proposed half integrators based on 2nd and 3rd order optimized DIs with existing $[2,17]$ models.

optimized solutions of 2nd and 3rd order DIs are as follows: particle range $=(-1,1)$, learning factors $\left(c_{1}\right.$ and $\left.c_{2}\right)=2$, diversity factor $=1$, maximum velocity factor $=0.5$, swarm size $=200$, and number of generations $=200$. The flowchart of modified PSO has been given in Figure 1 .

Intel Core 2 Duo CPU T6600@2.20 GHz (Installed Memory (RAM) of $4 \mathrm{~GB}$ ) has been used for simulations. Transfer functions of worst and average cases of $2 \mathrm{nd}$ and $3 \mathrm{rd}$ order DIs have been given in Tables 1 and 2, respectively, along with their corresponding fitness function values (F.V.).

In this paper when the code was run in $\mathrm{C}++$ for 100 iterations, the best optimized (with least mean square error) results for 2nd and 3rd order proposed DIs have been observed for 200 generations using 200 particles. Transfer functions (TFs) of proposed optimized integrators have been given next in pole zero form in (12) and (13), respectively.

TF of proposed 2nd order optimized integrator is

$$
H_{\mathrm{PSO} \_2 n d \_o p t}(z)=\left(\frac{(0.8651(z+0.1042)(z+0.6276))}{((z-1)(z+0.5547))}\right) \text {. }
$$

TF of proposed 3rd order optimized integrator is

$$
\begin{aligned}
& H_{\text {PSO_3rd_opt }}(z) \\
& \quad=\left(\frac{(0.8656(z+0.1057)(z+0.2021)(z+0.503))}{((z+0.38)(z+0.2522)(z-0.9997))}\right) .
\end{aligned}
$$

Proposed 2nd and 3rd order DIs optimized here by PSO have been compared with the recently published integrators optimized by different optimization algorithms, namely, LP 


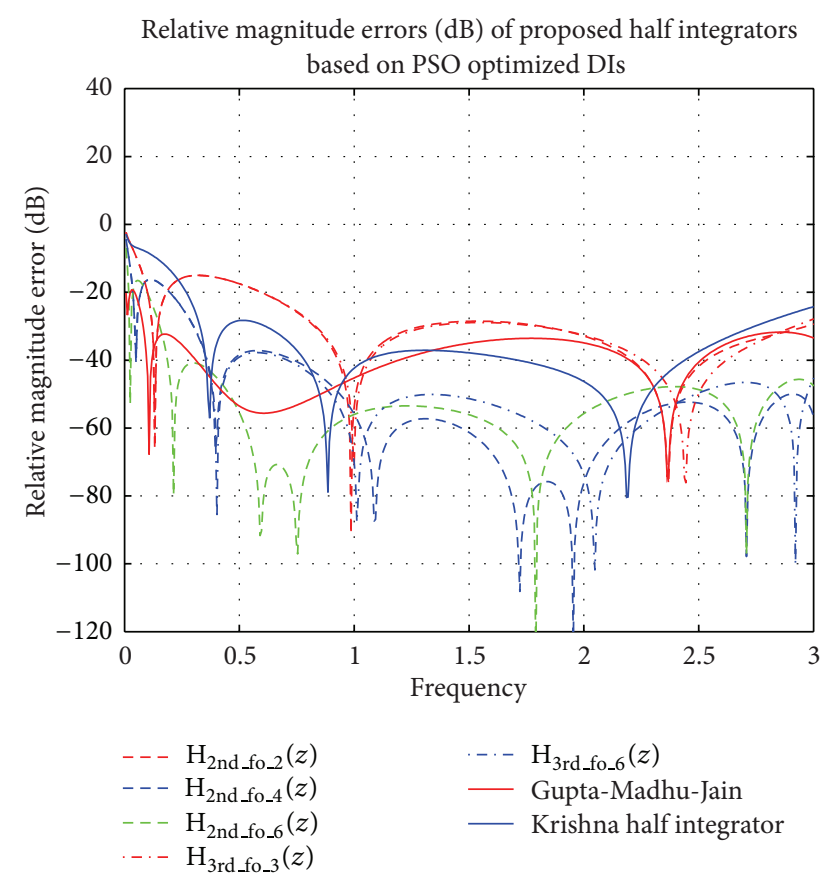

FIGURE 6: Comparison of RME values of proposed half integrators based on 2nd and 3rd order optimized DIs with existing [2, 17] models.

[34], GA [35], SA [39], and PZ [41], for validating efficient performance of proposed PSO. Comparisons of bode diagrams (i.e., magnitude versus the frequency $(w)$ and phase (degrees) versus frequency $(w)$ ) and RME responses (in $\mathrm{dB}$ ) have been presented in Figures 2, 3 and 4 respectively.

Results for performance values (RME) for each optimization method have been compared in Table 3. It can be observed from Figure 3 that the proposed PSO method clearly outperforms existing SA [39] and LP [34] techniques and gives comparable results for PZ [41] and GA [35] methods. The 2nd order DI, namely, $H_{\text {PSO_nd_opt }}(z)$ given in (12), outperforms Al-Alaoui 3-segment and 4-segment optimized DIs in complete spectrum. $H_{\text {PSO_2nd_opt }}(z)$ excels Jain-GuptaJain DI [35] in frequency range over $0 \leq \omega \leq 0.5 \pi$ radians and $2.58 \leq \omega \leq 3 \pi$ radians. PSO optimized proposed 3rd order DI, namely, $H_{\text {PSO_3rd_opt }}(z)$, clearly outperforms the existing Al-Alaoui optimized 3-segment, 4-segment [39], and Upadhyay [41] DIs in almost complete range with RME of $\leq$ $-41 \mathrm{~dB}$. $H_{\mathrm{PSO} \_3 r d o p t}(z)$ given in (13) gives comparable results with GA optimized [35] operator. Proposed optimized DIs are observed to satisfy stability criterion and show linearly decreasing phase responses (see Figure 4).

Proposed DIs optimized by PSO clearly excel operators optimized by SA [39], LP [34], and PZ [41] techniques, but lie in close vicinity of the GA [35] responses. So a statistical test has been done for comparing performances of PSO and GA for validating superiority of the proposed modified algorithm. The products of number of particles and number of generations $\left(N_{p} * N_{g}\right)$ have been varied from $(50 * 50)$ to $(100 * 100)$ and then to $(200 * 200)$ for different iterations and values of mean, median, and standard deviation have

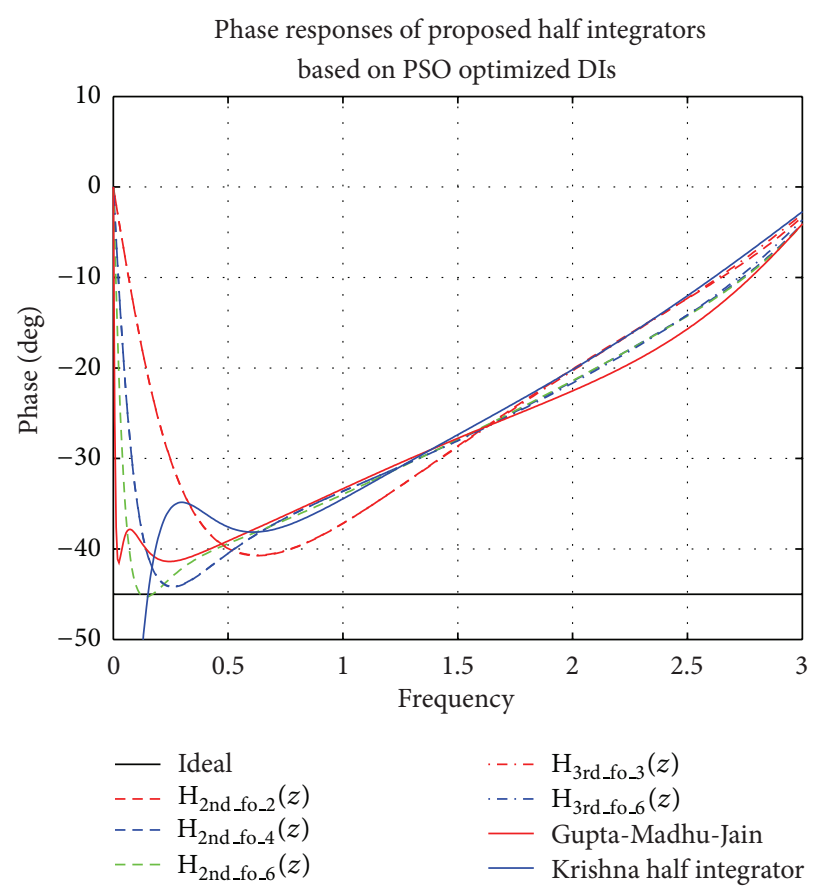

FIGURE 7: Comparison of phase responses of proposed half integrators based on 2nd and 3rd order optimized DIs with existing [2, 17] models.

been registered for all the worst, average, and best results of 2nd and 3rd order optimized DIs (see Table 4). From this table it is clear that PSO technique performs better than GA method and also it was noticed that with the increase in values of $N_{p}, N_{g}$, and number of iterations, the response of resultant DIs comes closer to the ideal response as mean, median, and standard deviation values go on decreasing with efficient minimization of error function. In this paper we have considered values of root mean square error.

\section{Discretization of \\ Proposed 2nd and 3rd Order Optimized DIs for Half Integrator Models}

The optimized integer order DIs derived in Section 2 are used as $s$-to- $z$ transformations for CFE based indirect discretization [2, 23, 24] scheme. Formula given by Khovanskii in [29] has been used for different rational approximations of FOIs. That is

$$
\begin{aligned}
(s)^{ \pm \alpha}= & \frac{1}{1-} \frac{\alpha s}{1+(1+\alpha) s^{-}} \frac{(1+\alpha) s(1+s)}{2+(3+\alpha) s^{-}} \frac{2(2+\alpha) s(1+s)}{3+(5+\alpha) s^{-}} \\
& \times \frac{3(3+\alpha) s(1+s)}{4+(9+\alpha) s^{-}} \frac{4(4+\alpha) s(1+s)}{5+(9+\alpha) s^{-}} \\
& \times \frac{5(5+\alpha) s(1+s)}{6+(11+\alpha) s^{-}} \frac{6(6+\alpha) s(1+s)}{7+(13+\alpha) s^{-}}
\end{aligned}
$$

Infinite series of (14) can be terminated up to 2, 4, and 6 terms, and CFE based indirect discretization used in 


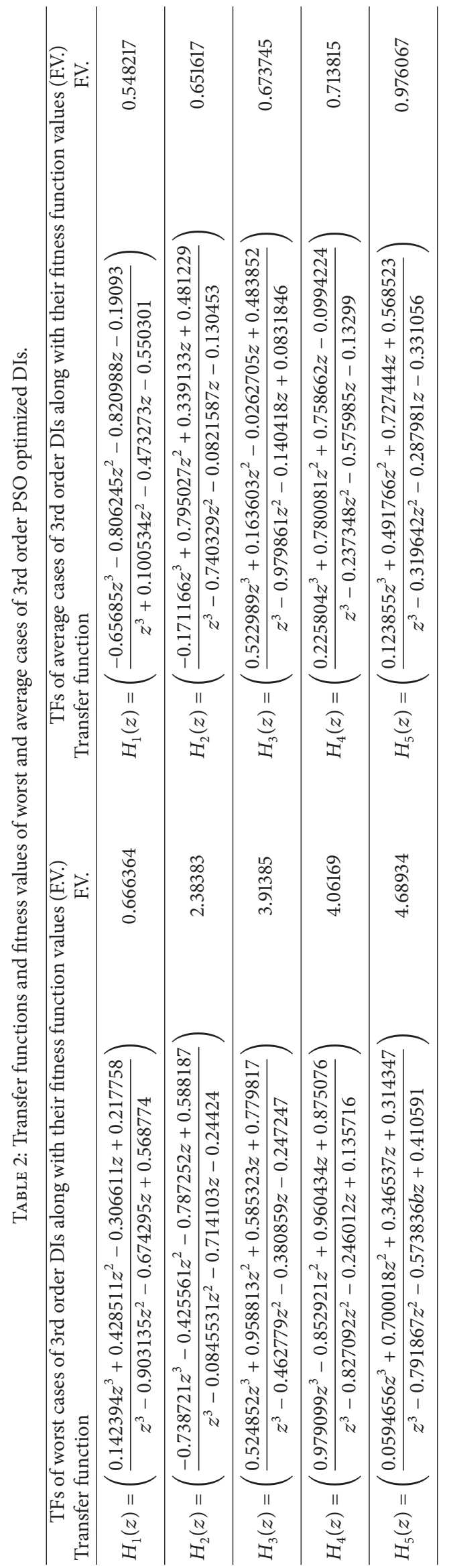


TABLE 3: Comparisons of RME values of the proposed PSO optimized DIs with other integer order existing integrators optimized by different optimization techniques.

\begin{tabular}{|c|c|c|c|c|c|c|c|}
\hline$\omega(\pi \mathrm{rad})$ & $\begin{array}{c}\text { Al-Alaoui } \\
\text { 3-segment } \\
\text { (SA) }\end{array}$ & $\begin{array}{c}\text { Al-Alaoui } \\
\text { 4-segment } \\
\text { (SA) }\end{array}$ & $\begin{array}{l}\text { Jain-Gupta-Jain } \\
\text { (GA) }\end{array}$ & $\begin{array}{l}\text { Upadhyay } \\
(\mathrm{P}-\mathrm{Z})\end{array}$ & $\begin{array}{c}\text { Gupta-Jain-Kumar } \\
\text { (LP) }\end{array}$ & $\begin{array}{l}\text { Proposed } \\
\text { 2nd order } \\
\text { DI (PSO) }\end{array}$ & $\begin{array}{l}\text { Proposed } \\
\text { 3rd order } \\
\text { DI (PSO) }\end{array}$ \\
\hline 0.25 & -72.03 & -60.18 & -54.46 & -48.31 & -71.06 & -72.03 & -63.82 \\
\hline 0.5 & -49.15 & -41.21 & -59.47 & -51.65 & -59.89 & -59.68 & -73.04 \\
\hline 0.75 & -37.25 & -34.02 & -80.04 & -61.71 & -63.91 & -53.27 & -71.45 \\
\hline 1.0 & -30.29 & -30.34 & -57.28 & -57.28 & -49.94 & -49.88 & -64.63 \\
\hline 1.25 & -22.72 & -32.96 & -53.84 & -48.71 & -39.33 & -48.71 & -68.2 \\
\hline 1.75 & -11.64 & -35.05 & -53.85 & -47.91 & -31.46 & -62.62 & -54.2 \\
\hline 2.0 & -8.24 & -19.11 & -60.53 & -52.89 & -31.43 & -49.98 & -49.94 \\
\hline 2.25 & -6.92 & -15.99 & -53.84 & -61.5 & -34.5 & -42.77 & -53.66 \\
\hline 2.75 & -18.19 & -19.73 & -37.54 & -37.76 & -45.88 & -55.55 & -40.61 \\
\hline 3.0 & -4.17 & -18.11 & -35.52 & -36.44 & -30.14 & -41.34 & -45.01 \\
\hline
\end{tabular}

TABLE 4: Statistical test for comparison of proposed PSO and GA techniques for approximations of 2nd and 3rd order digital integrators.

\begin{tabular}{|c|c|c|c|c|c|c|}
\hline & \multicolumn{3}{|c|}{ Genetic algorithm } & \multicolumn{3}{|c|}{ Modified PSO algorithm } \\
\hline \multicolumn{7}{|c|}{ Approximating 2nd order digital integrator for comparing PSO and GA } \\
\hline$N_{p} * N_{g}$ & $50 * 50$ & $100 * 100$ & $200 * 200$ & $50 * 50$ & $100 * 100$ & $200 * 200$ \\
\hline Mean & 0.915405 & 0.230096 & 0.0874599 & 0.614370 & 0.201256 & 0.0936231 \\
\hline Median & 0.482815 & 0.194402 & 0.075695 & 0.491575 & 0.114377 & 0.0488172 \\
\hline Standard deviation & 0.737526 & 0.131617 & 0.0400587 & 0.43579 & 0.137471 & 0.042671 \\
\hline \multicolumn{7}{|c|}{ Approximating 3rd order digital integrator for comparing PSO and GA } \\
\hline$N_{p} * N_{g}$ & $50 * 50$ & $100 * 100$ & $200 * 200$ & $50 * 50$ & $100 * 100$ & $200 * 200$ \\
\hline Mean & 0.915406 & 0.230096 & 0.0874599 & 0.633554 & 0.221398 & 0.0969881 \\
\hline Median & 0.1478565 & 0.069402 & 0.02937085 & 0.1801123 & 0.1207528 & 0.0861992 \\
\hline Standard deviation & 0.737526 & 0.131617 & 0.0400587 & 0.491451 & 0.152073 & 0.0530145 \\
\hline
\end{tabular}

[24] has been adopted for deriving half integrators fitted in continuous-time domain (see Table 5). Also 2nd order optimized DI. resulted in 2nd, 4 th, and 6th order FOIs whereas 3rd order optimized DI when substituted for different terms resulted in 3rd and 6th order half integrators. In the TFs of derived proposed half integrators given in Table 5 , order of optimized DIs and order of FOIs are used as subscripts of symbol " $H$ ".

\section{Comparisons of Proposed FOIs Based on 2nd and 3rd Order Optimized DIs with the Existing Models}

Simulation results of comparison of magnitude responses, RME and phase responses of half integrators (see Table 5) based on different 2 nd and 3rd order optimized DIs (given in (12) and (13)) with the existing half integrators given in [2, 17] and ideal half integrator have been presented in Figures 5-7.

Comparison of RME values of proposed and existing FOIs has been presented in Table 6. It is observed from Figure 6 that the proposed 2nd and 3rd order FOIs, namely, $H_{2 \text { nd_fo_2 }}(z)$ and $H_{3 \text { rd_fo_3 } 3}(z)$, only lag in performance but still these outperform the existing $[2,17]$ models in higher frequency spectrum. The 6 th order operator $H_{2 \text { nd_fo_ } 6}(z)$ is the best among the proposed FOIs and it clearly outperforms all the existing models $[2,17]$ in complete frequency spectrum with RME of $\leq-40 \mathrm{~dB}$. Proposed $H_{2 \text { nd_fo_2 }_{2}}(z)$ and $H_{3 \text { rd_fo_3 }_{3}}(z)$ outperform Gupta-Madhu-Jain half integrator [17] in frequency range over $0.88 \leq \omega \leq 3 \pi$ radians. $H_{2 \text { nd_fo_ } 4}(z)$, $H_{2 \text { nd_fo_6 }}(z)$, and $H_{3 \text { rd_fo_6 }}(z)$ half integrators excel Krishna half integrator [2] in almost complete range. All the proposed FOIs are observed to be stable and follow linear phase curve in almost complete frequency spectrum (see Figure 7).

\section{Conclusions}

This paper attempts to focus on a simple yet unaddressed fact which till date has remained in the backdrop as the optimization of an integer order operator before converting it into its fractional the order (fo) counterparts. Proposed FOIs validate that properties of an efficiently optimized DI pass directly into its fractional domain when it is used as $s$ to- $z$ transformation during indirect discretization. Here, 2nd and 3rd order integer order integrators have been explored by PSO algorithm for finding their optimal solutions for a minimized error fitness function. The simulation results of integer order operators have revealed the effectiveness of the proposed modified PSO algorithm, because of their low 
TABLE 5: Mathematical models of proposed half integrators based on 2nd and 3rd order PSO optimized DIs.

Mathematical models of half integrators based on 2nd and 3rd order PSO optimized DIs Name of PSO optimized DIs

Transfer functions of FOIs derived by indirect discretization of CFE

\begin{tabular}{|c|c|}
\hline$H_{\text {PSO_2nd_opt }}(z)$ & $\begin{array}{c}H_{\text {2nd-fo-2 }}(z)=\left(\frac{0.9302(z-0.1825)(z+0.5869)}{(z-0.7422)(z+0.5604)}\right) \\
H_{\text {2nd-fo-4 }}(z)=\left(\frac{0.93011(z-0.003372)(z+0.5633)(z+0.6071)(z-0.6396)}{(z-0.9034)(z-0.293)(z+0.5788)(z+0.5566)}\right) \\
H_{\text {2nd-fo-6 }}(z)=\left(\frac{\left(\begin{array}{c}0.93011(z-0.8073)(z-0.3427)(z+0.616) \\
(z+0.5758)(z+0.5587)(z+0.04899)\end{array}\right)}{\left(\begin{array}{c}(z+0.5936)(z+0.5649)(z+0.5556) \\
(z-0.5924)(z-0.9502)(z-0.1107)\end{array}\right)}\right)\end{array}$ \\
\hline$H_{\text {PSO_3rd_opt }}(z)$ & $\begin{array}{r}H_{3 \text { rd-fo-3 }}(z)=\left(\frac{0.93046(z-0.1852)(z+0.2354)(z+0.433)}{(z-0.742)(z+0.3892)(z+0.2492)}\right) \\
H_{3 \text { rd-fo-6 }}(z)=\left(\frac{\left(\begin{array}{c}0.93038(z-0.6395)(z+0.4678)(z+0.3939) \\
(z+0.2476)(z+0.223)(z-0.008033)\end{array}\right)}{\left(\begin{array}{c}(z-0.9031)(z-0.2946)(z+0.2512) \\
(z+0.2397)(z+0.383)(z+0.4194)\end{array}\right)}\right.\end{array}$ \\
\hline
\end{tabular}

TABLE 6: Comparisons of RME values of the proposed half integrators based on optimized DIs with the existing models.

\begin{tabular}{|c|c|c|c|c|c|c|c|}
\hline \multirow[t]{2}{*}{$\begin{array}{l}\omega \\
(\pi \mathrm{rad})\end{array}$} & \multicolumn{3}{|c|}{$\begin{array}{c}\text { Proposed half integrators based on 2nd order } \\
\text { PSO optimized DI }\end{array}$} & \multicolumn{2}{|c|}{$\begin{array}{l}\text { Proposed half integrators based on 3rd } \\
\text { order PSO optimized DI }\end{array}$} & \multirow{2}{*}{$\begin{array}{l}\text { Gupta-Madhu- } \\
\text { Jain half } \\
\text { integrator }\end{array}$} & \multirow[t]{2}{*}{$\begin{array}{c}\text { Krishna half } \\
\text { integrator }\end{array}$} \\
\hline & $H_{2 \text { nd-fo-2 }}(z)$ & $H_{\text {2nd-fo-4 }}(z)$ & $H_{2 \text { nd-fo- } 6}(z)$ & $H_{3 \text { rd-fo- } 3}(z)$ & $H_{3 \text { rd-fo- } 6}(z)$ & & \\
\hline 0.25 & -15.79 & -24.55 & -43.25 & -15.79 & -29.26 & -35.53 & -17.42 \\
\hline 0.5 & -17.52 & -38.74 & -55.85 & -17.52 & -38.97 & -53.62 & -28.31 \\
\hline 0.75 & -25.82 & -41.56 & -85.14 & -25.81 & -42.61 & -53.65 & -35.56 \\
\hline 1.0 & -58.3 & -58.3 & -55.44 & -64.01 & -74.75 & -45.26 & -42.05 \\
\hline 1.25 & -31.25 & -57.34 & -53.48 & -31.13 & -50.67 & -38.93 & -37.13 \\
\hline 1.75 & -28.94 & -81.15 & -75.32 & -29.82 & -58.25 & -33.59 & -40.57 \\
\hline 2.0 & -32.62 & -75.83 & -55.54 & -32.62 & -73.25 & -34.88 & -46.46 \\
\hline 2.25 & -41.43 & -57.13 & -48.99 & -41.15 & -58.93 & -43.69 & -55.28 \\
\hline 2.75 & -32.99 & -59.6 & -59.6 & -36.33 & -46.94 & -32.6 & -29.8 \\
\hline 3.0 & -29.56 & -56.03 & -47.75 & -28.27 & -46.74 & -33.74 & -24.3 \\
\hline
\end{tabular}

orders and very less magnitude errors as compared to existing ones. Proposed FOIs based on the optimized DIs present very less RME values of the order of $-40 \mathrm{~dB}$ and show linear phase curves in almost full band of Nyquist frequency.

\section{References}

[1] L. Debnath, "Recent applications of fractional calculus to science and engineering," International Journal of Mathematics and Mathematical Sciences, vol. 2003, no. 54, pp. 3413-3442, 2003.

[2] B. T. Krishna, "Studies on fractional order differentiators and integrators: a survey," Signal Processing, vol. 91, no. 3, pp. 386426, 2011.

[3] N. M. F. Ferreira, F. B. Duarte, M. F. M. Lima, M. G. Marcos, and J. A. T. Machado, "Application of fractional calculus in the dynamical analysis and control of mechanical manipulators," Fractional Calculus and Applied Analysis, vol. 11, pp. 91-113, 2008.
[4] S. Manabe, "The noninteger integral and its application to control systems," English Translation Journal Japan, vol. 6, pp. 83-87, 1961.

[5] J. A. T. Machado and A. M. S. Galhano, "A new method for approximating fractional derivatives: application in nonlinear control," in Proceedings of the 6th EUROMECH Nonlinear Dynamics Conference (ENOC '08), pp. 4-8, Saint Petersburg, Russia, July 2008.

[6] Y. Ferdi, J. P. Herbeuval, A. Charef, and B. Boucheham, "R wave detection using fractional digital differentiation," ITBM-RBM, vol. 24, no. 5-6, pp. 273-280, 2003.

[7] J. Mocak, I. Janiga, M. Rievaj, and D. Bustin, "The use of fractional differentiation or integration for signal improvement," Measurement Science Review, vol. 7, pp. 39-42, 2007.

[8] B. Mathieu, P. Melchior, A. Oustaloup, and C. Ceyral, "Fractional differentiation for edge detection," Signal Processing, vol. 83, no. 11, pp. 2421-2432, 2003.

[9] Y. Pu, W. Wang, J. Zhou, Y. Wang, and H. Jia, "Fractional differential approach to detecting textural features of digital 
image and its fractional differential filter implementation," Science in China F, vol. 51, no. 9, pp. 1319-1339, 2008.

[10] S. Das, I. Pan, S. Das, and A. Gupta, "Improved model reduction and tuning of fractional-order $\mathrm{PI}^{\lambda} \mathrm{D}^{\mu}$ controllers for analytical rule extraction with genetic programming," ISA Transactions, vol. 51, no. 2, pp. 237-261, 2012.

[11] S. Das, I. Pan, K. Halder, S. Das, and A. Gupta, "Impact of fractional order integral performance indices in LQR based PID controller design via optimum selection of weighting matrices," in Proceedings of the International Conference on Computer Communication and Informatics (ICCCI '12), pp. 1-6, Coimbatore, India, January 2012.

[12] Y. Chen and B. M. Vinagre, "A new IIR-type digital fractional order differentiator," Signal Processing, vol. 83, no. 11, pp. 23592365, 2003.

[13] Y. Chen, B. M. Vinagre, and I. Podlubny, "Continued fraction expansion approaches to discretizing fractional order derivatives-an expository review," Nonlinear Dynamics, vol. 38, no. 1-4, pp. 155-170, 2004.

[14] Y. Q. Chen and K. L. Moore, "Discretization schemes for fractional-order differentiators and integrators," IEEE Transactions on Circuits and Systems I, vol. 49, no. 3, pp. 363-367, 2002.

[15] M. A. Al-Alaoui, "Novel class of digital integrators and differentiators," IEEE Transactions on Signal Processing, p. 1, 2009.

[16] M. A. Al-Alaoui, "Al-Alaoui operator and the new transformation polynomials for discretization of analogue systems," Electrical Engineering, vol. 90, no. 6, pp. 455-467, 2008.

[17] M. Gupta, M. Jain, and N. Jain, "A new fractional order recursive digital integrator using continued fraction expansion," in Proceedings of the India International Conference on Power electronics (IICPE), pp. 1-3, New Delhi, India, 2010.

[18] M. Gupta, P. Varshney, and G. S. Visweswaran, "Digital fractional-order differentiator and integrator models based on first-order and higher order operators," International Journal of Circuit Theory and Applications, vol. 39, no. 5, pp. 461-474, 2011.

[19] F. Leulmi and Y. Ferdi, "An improvement of the rational approximation of the fractional operator $s^{\alpha}$," in Proceedings of the Saudi International Electronics, Communications and Photonics Conference (SIECP '11), pp. 1-6, Riyadh, Saudi Arabia, 2011.

[20] Y. Ferdi, "Computation of fractional order derivative and integral via power series expansion and signal modelling," Nonlinear Dynamics, vol. 46, no. 1-2, pp. 1-15, 2006.

[21] G. S. Visweswaran, P. Varshney, and M. Gupta, "New approach to realize fractional power in $\mathrm{z}$-domain at low frequency," IEEE Transactions on Circuits and Systems II, vol. 58, no. 3, pp. 179183, 2011.

[22] P. Varshney, M. Gupta, and G. S. Visweswaran, "Switched capacitor realizations of fractional-order differentiators and integrators based on an operator with improved performance," Radioengineering, vol. 20, no. 1, pp. 340-348, 2011.

[23] B. T. Krishna and K. V. V. Reddy, "Design of fractional order digital differentiators and integrators using indirect discretization," An International Journal for Theory and Applications, vol. 11, pp. 143-151, 2008.

[24] B. T. Krishna and K. V. V. Reddy, "Design of digital differentiators and integrators of order 1/2," World Journal of Modelling and Simulation, vol. 4, pp. 182-187, 2008.

[25] R. Yadav and M. Gupta, "Design of fractional order differentiators and integrators using indirect discretization approach," in Proceedings of the 2nd International Conference on Advances in Recent Technologies in Communication and Computing (ARTCom '10), pp. 126-130, Kottayam, India, October 2010.

[26] R. Yadav and M. Gupta, "Design of fractional order differentiators and integrators using indirect discretization scheme," in Proceedings of the India International Conference on Power Electronics (IICPE '10), New Delhi, India, January 2011.

[27] G. E. Carlson and C. A. Halijak, "Simulation of the fractional derivative operator $(1 / s)^{1 / 2}$ and the fractional integral operator $(1 / s)^{1 / 2}$," Kansas State University Bulletin, vol. 45, pp. 1-22, 1961.

[28] S. K. Steiglitz, "Computer-aided design of recursive digital filters," IEEE Transactions Audio and Electroacoustics, vol. AU18, no. 2, pp. 123-129, 1970.

[29] A. N. Khovanskii, The Application of Continued Fractions and their Generalizations to Problems in Approximation Theory, $\mathrm{P}$. Noordhoff Ltd., 1963, Translate by Peter Wynn.

[30] S. C. Roy, "Some exact steady-state sinewave solutions of nonuniform RC lines," British Journal of Applied Physics, vol. 14, pp. 378-380, 1963.

[31] A. Oustaloup, F. Levron, B. Mathieu, and F. M. Nanot, "Frequency-band complex noninteger differentiator: characterization and synthesis," IEEE Transactions on Circuits and Systems I, vol. 47, no. 1, pp. 25-39, 2000.

[32] G. Maione, "Laguerre approximation of fractional systems," Electronics Letters, vol. 38, no. 20, pp. 1234-1236, 2002.

[33] N. Papamarkos and C. Chamzas, "A new approach for the design of digital integrators," IEEE Transactions on Circuits and Systems I, vol. 43, no. 9, pp. 785-791, 1996.

[34] M. Gupta, M. Jain, and B. Kumar, "Recursive wideband digital integrator and differentiator," International Journal of Circuit Theory and Applications, vol. 39, no. 7, pp. 775-782, 2011.

[35] N. Jain, M. Gupta, and M. Jain, "Linear phase second order recursive digital integrators and differentiators," Radioengineering Journal, vol. 21, pp. 712-717, 2012.

[36] C.-C. Hse, W.-Y. Wang, and C.-Y. Yu, "Genetic algorithmderived digital integrators and their applications in discretization of continuous systems," in Proceedings of the CEC Congress on Evolutionary Computation, pp. 443-448, Honolulu, Hawaii, USA, 2002.

[37] S. Das, B. Majumder, A. Pakhira, I. Pan, S. Das, and A. Gupta, "Optimizing continued fraction expansion based iir realization of fractional order differ-integrators with genetic algorithm," in Proceedings of the International Conference on Process Automation, Control and Computing (PACC '11), pp. 1-6, Coimbatore, India, July 2011.

[38] G. K. Thakur, A. Gupta, and D. Upadhyay, "Optimization algorithm approach for error minimization of digital integrators and differentiators," in Proceedings of the International Conference on Advances in Recent Technologies in Communication and Computing (ARTCom '09), pp. 118-122, Kottayam, India, October 2009.

[39] M. A. Al-Alaoui, "Class of digital integrators and differentiators," IET Signal Processing, vol. 5, no. 2, pp. 251-260, 2011.

[40] D. K. Upadhyay and R. K. Singh, "Recursive wideband digital differentiator and integrator," Electronics Letters, vol. 47, no. 11, pp. 647-648, 2011.

[41] D. K. Upadhyay, "Class of recursive wide band digital differentiators and integrators," Radioengineering Journal, vol. 21, no. 3, pp. 904-910, 2012.

[42] J. Kennedy and R. Eberhart, "Particle swarm optimization," in Proceedings of the IEEE International Conference on Neural Networks, pp. 1942-1948, December 1995. 
[43] Y.-T. Kao and E. Zahara, "A hybrid genetic algorithm and particle swarm optimization for multimodal functions," Applied Soft Computing Journal, vol. 8, no. 2, pp. 849-857, 2008.

[44] Y. Shi and R. C. Eberhart, "Empirical study of particle swarm optimization," in Proceedings of the IEEE international Conference Evolution Computer, pp. 1945-1950, Washington, DC, USA, 1999. 


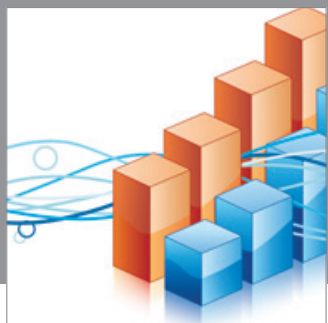

Advances in

Operations Research

mansans

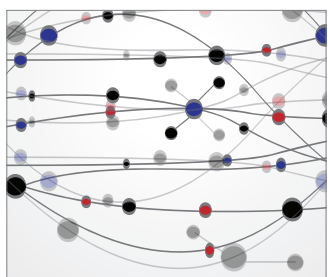

The Scientific World Journal
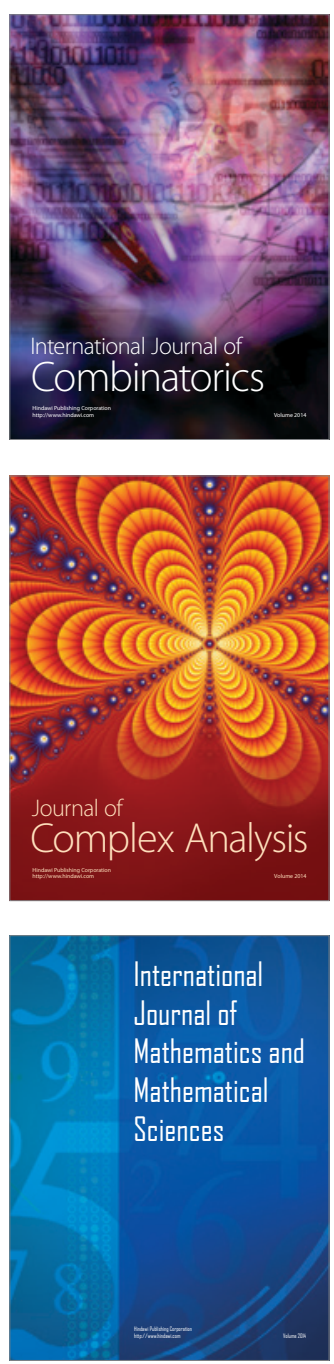
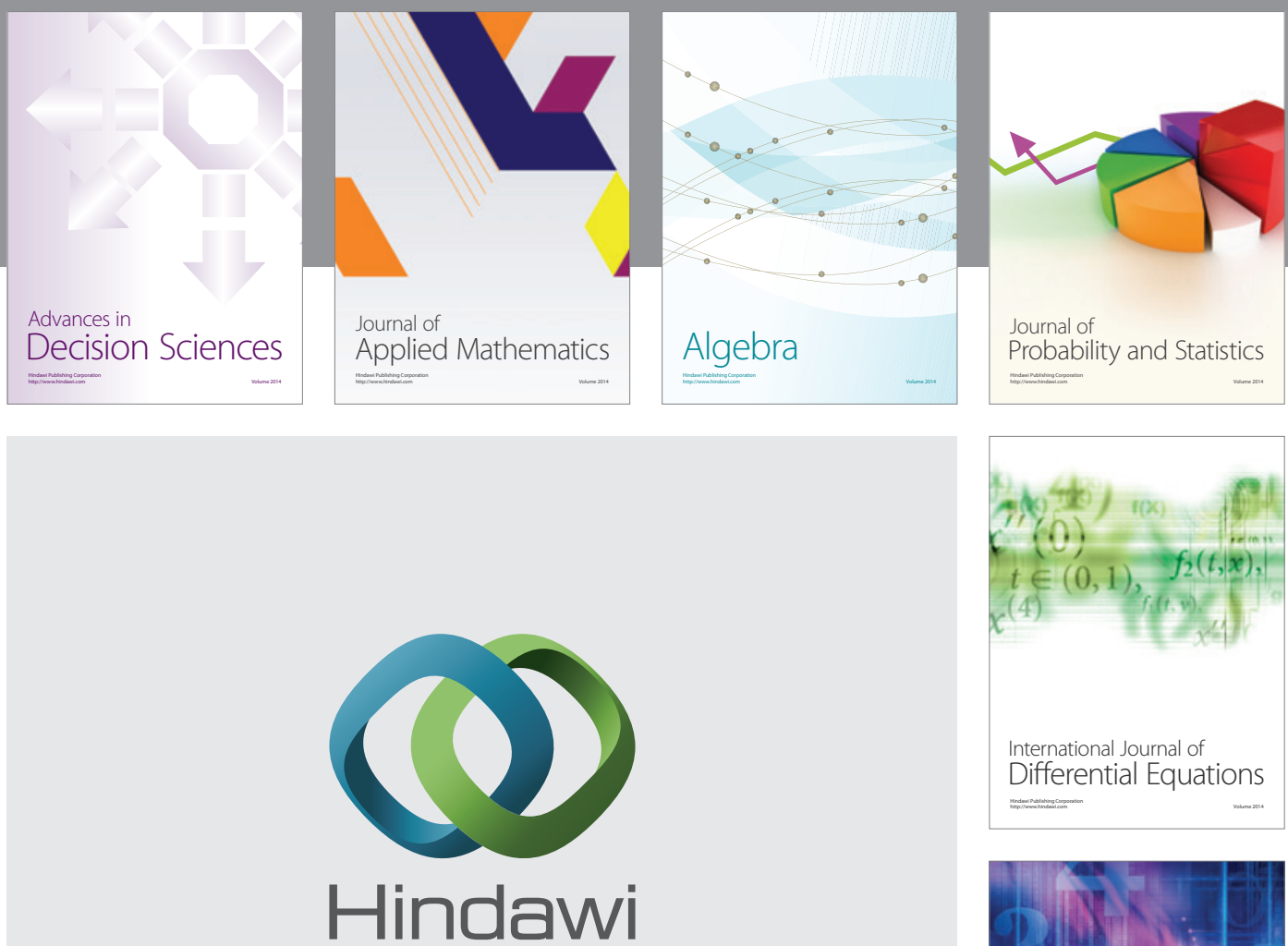

Submit your manuscripts at http://www.hindawi.com
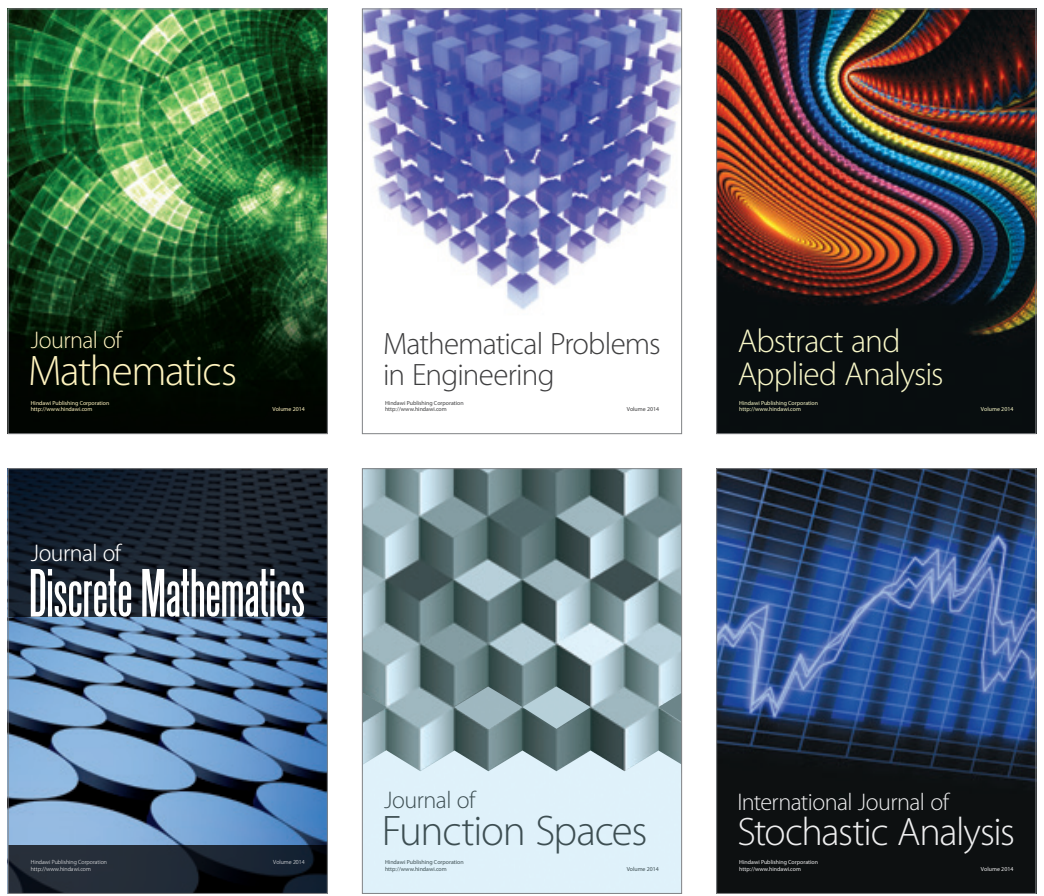

Journal of

Function Spaces

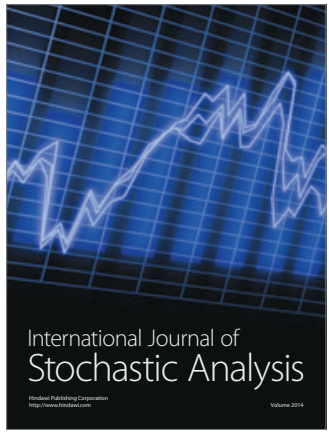

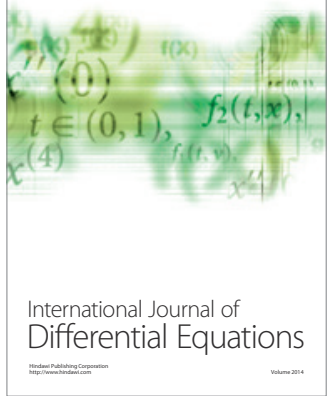
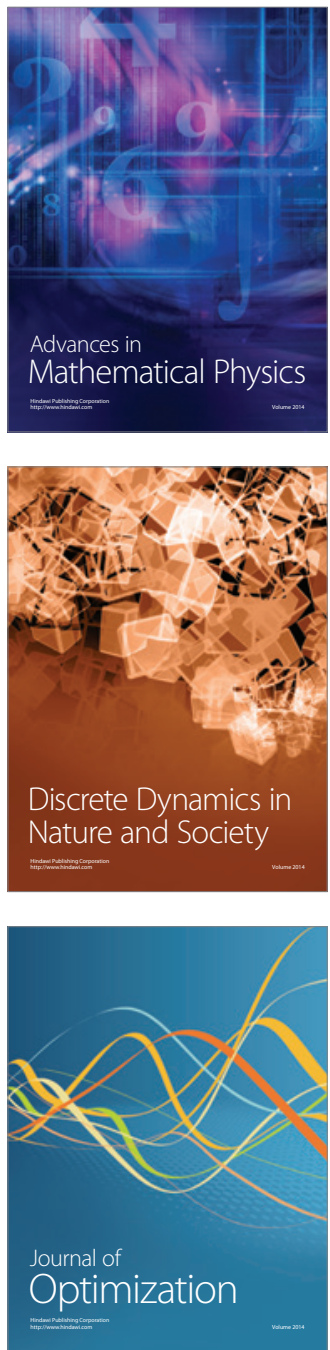